\title{
SENSITIVITY OF TIME-DIVISION MULTIPLEXING PARALLEL A/D CONVERTERS TO ANALOG IMPERFECTION
}

\author{
Davud Asemani and Jacques Oksman \\ Supelec, Department of signal processing and electronic systems \\ 3, Rue Joliot Curie, Gif-sur-Yvette, 91192, France \\ Email: firstname.lastname@Supelec.fr
}

\begin{abstract}
In this paper, the Time-Division Multiplexing (TDM) architecture for the Hybrid Filter Bank (HFB) A/D Converters (ADCs) is studied in the time domain. Giving a brief survey on the TDM structure, the classical and TDM HFB-based ADCs are compared in terms of the output resolution for some input signals. To study the sensitivity to the realization errors, both structures are simulated assuming the same realization errors in the analysis filter banks. The TDM HFBbased ADC exhibits a better performance either in the presence or in the absence of realization errors than the classical one. Besides, the input-output relationship is demonstrated to be Linear Time-Invariant (LTI) for the TDM HFB, but it is non-LTI in the the classical HFB case. Thus, it is possible only in the TDM case that a blind deconvolution method is employed for adaptively compensating the realization errors.
\end{abstract}

Index Terms - Hybrid Filter Bank, Time-division Multiplexing, A/D converter, Software-Defined Radio systems

\section{INTRODUCTION}

The important challenge in $\mathrm{A} / \mathrm{D}$ and $\mathrm{D} / \mathrm{A}$ conversion is to achieve both factors of high-speed and high-resolution conversion at the same time. The delta-sigma $(\Delta \Sigma)$ converters may provide the best resolution, but are so limited in the band-width of conversion [1]. The demand for A/D or D/A converters with higher speeds has dramatically increased for realizing the new communications concepts such as Software-Defined Radio (SDR) approach which will form a new industry on an even larger scale than the personal computer industry [2]. Putting the high-precision ADCs in Parallel, a wide-band ADC may be obtained. The timeinterleaving and discrete-time HFB structures have already been proposed in this regard. They encounter nevertheless with the problems of an extremely-high sensitivity to the mismatch of converters and practical speed limitation respectively [3]. The continuous-time HFB structure using analog analysis filters has been proposed as a suitable candidate for realizing the wide-band ADCs. Figure 1 shows the classical HFB structure for $\mathrm{A} / \mathrm{D}$ conversion where $M$ and $T$ are associated with the number of branches and Nyquist sampling period respectively [4]. Employing this parallel

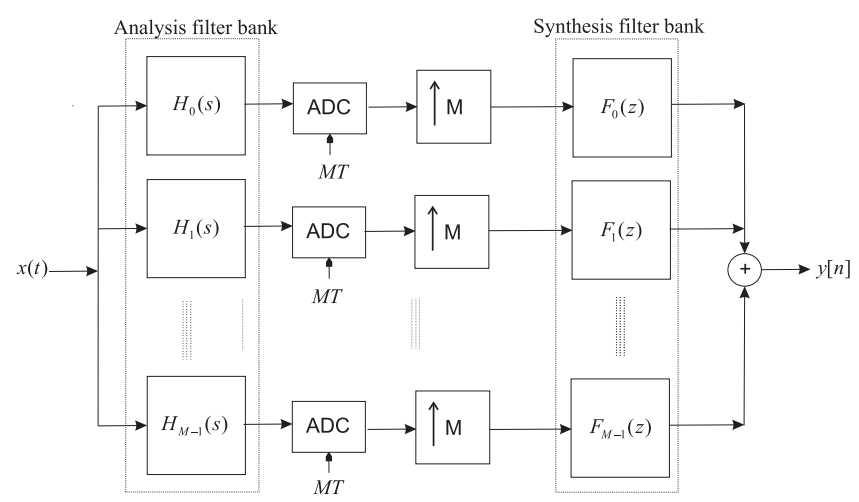

Fig. 1. The classical HFB-based A/D converter.

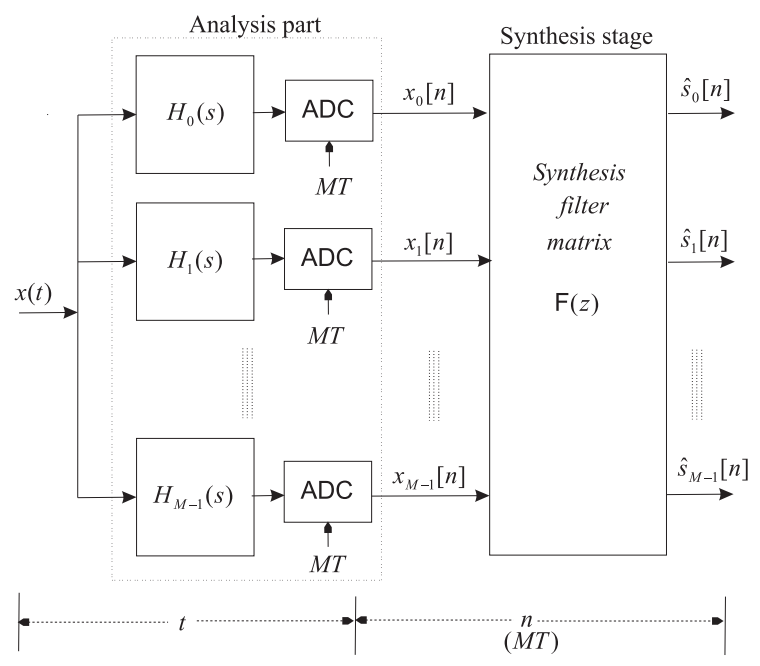

Fig. 2. The TDM architecture of HFB-based ADC for estimating the TDM components of the input signal. The outputs $\hat{s}_{0}[n], \hat{s}_{1}[n], \ldots$, and $\hat{s}_{M-1}[n]$ are the estimated TDM signals.

structure, $M \mathrm{~A} / \mathrm{D}$ converters are now used in parallel working at $\frac{1}{M T}$ whereas the original analog input is supposed to be limited to the frequency interval $\left[-\frac{\pi}{T}, \frac{\pi}{T}\right]$. An $M$ - 
branch HFB ADC exhibits $M-1$ interference terms called aliasing at the output which restrict the output resolution like the quantization noise. The analog HFB-based A/D converters have exhibited a good performance (low aliasing terms) using simply-realizable first- and second-order analysis filters and FIR digital synthesis filters if a small ratio of oversampling is considered. However, the related performance in the presence of even small realization errors degrades so much [5]. It is then necessary to somehow mitigate or compensate the realization errors for having a useful HFB-based ADC. Digital techniques have been dealt with for managing the problem of high sensitivity to the realization errors. However, the proposed methods are limited to some specific errors or cases [6]. Pinheiro et. al. tried to optimize the design of HFB structures in terms of realization errors [7], but their proposed solution does not include a compensation technique. They have only proposed a weighted criterion of distortion and aliasing terms which only leads to less than five $d B$ of improvement. Besides, this improvement is reported for the classical HFB-based ADC without any oversampling process. When no oversampling is used, the HFB structure is relatively robust versus realization errors [5], but the related performance is not acceptable for the practical applications unless a small oversampling ratio is considered.

The blind deconvolution techniques as a candidate for handling the sensitivity of HFB structures to the realization errors are applicable for LTI systems. However, the classical HFB architecture is associated with a time-variant relation between its input and output because of decimation process implied in the sampling at $\frac{1}{M T}$. Therefore, it is not possible to directly apply a blind deconvolution technique to the classical HFB ADC. A new HFB structure called TimeDivision Multiplexing (TDM) architecture has recently been offered that provides an LTI input-output relationship [8]. Figure 2 shows the TDM HFB-based ADC. It may be seen that a matrix $\mathbf{F}(z)$ of $M^{2}$ digital filters is considered in the synthesis stage for an $M$-branch HFB structure, instead of $M$ ones required for the classical one (compare figures 1 and 2). In the TDM HFB architecture, $M$ consecutive samples (at the Nyquist rate $\frac{1}{T}$ ) of the original input $x(t)$ are supposed as the input vector $\mathbf{s}[n]$ :

$$
\mathbf{s}[n]=\left[\begin{array}{c}
s_{0}[n] \\
s_{1}[n] \\
\vdots \\
s_{M-1}[n]
\end{array}\right]=\left[\begin{array}{c}
x\left(n^{\prime} T\right) \\
x\left(\left(n^{\prime}-1\right) T\right) \\
\vdots \\
x\left(\left(n^{\prime}-(M-1)\right) T\right)
\end{array}\right]_{n^{\prime}=n M}
$$

where $n$ and $n^{\prime}$ may be supposed as the discrete-time indices associated with the sampling rates $\frac{1}{M T}$ and $\frac{1}{T}$ respectively. The TDM HFB-based ADC tries to approximate the input vector $\mathbf{s}[n]$ at the output $\widehat{\mathbf{s}}[n]$. A blind deconvolution technique may be applied to the TDM HFB-based ADC to correct the analog imperfections, but this is not possible for the classical HFB. Moreover, the spectral simulations have shown that the TDM HFB-based ADC may result in a better performance than the classical one in the absence of realization errors [8]. In this paper, the TDM HFB-based ADC is simulated in the time-domain to demonstrate the validity of proposed model in terms of output resolution. The performance of the TDM HFB is also studied in the presence of realization errors for comparing with the one associated with the classical HFB. The respective sensitivities to the realization errors are compared as well. For this purpose, the TDM HFB architecture is briefly described and the related Perfect Reconstruction (PR) equations are presented in the next section II. Then, an eight-branch structure is assumed for simulating in the time domain for both the classical and TDM architectures. The related output resolutions and sensitivities to the analog imperfections are discussed in the section III. At last, the results of simulations and the comparison are summarized in the section of conclusion IV.

\section{TDM HFB ARCHITECTURE}

\section{II-A. MIMO model of TDM HFB ADC}

In the previous section, it was mentioned that the TDM architecture provides an Multiple-Input Multiple-Output (MIMO) structure for the parallel HFB-based ADC. For better following the TDM structure, a MIMO model has been proposed for the TDM HFB-based ADC so that the input vector $\mathbf{s}[n]$ is apparent [8]. Figure 3 shows this MIMO model

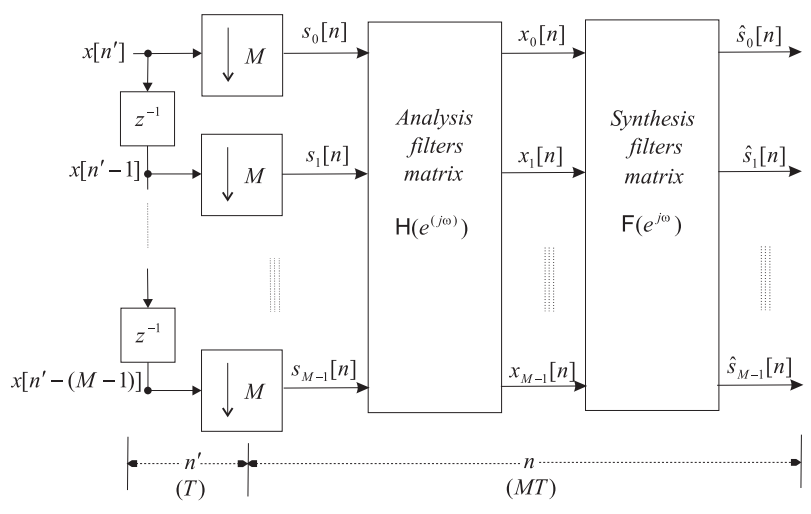

Fig. 3. The MIMO model of TDM HFB-based A/D converter using the TDM signals $s_{0}[n], s_{1}[n], \ldots$, and $s_{M-1}[n] . n^{\prime}$ and $n$ represent the discrete-time indices related to the rates $\frac{1}{T}$ and $\frac{1}{M T}$ respectively.

of TDM HFB-based ADC neglecting the quantization noise of ADCs. For convenience, the original analog input $x(t)$ has been replaced by $x\left[n^{\prime}\right]$ which is obtained by sampling $x(t)$ at $\frac{1}{T}\left(x\left[n^{\prime}\right]=x\left(n^{\prime} T\right)\right)$. It is desired to reconstruct the sequence $x\left[n^{\prime}\right]$ at the output. Invoking this MIMO model, the decimators exist no longer between the input $\mathbf{s}[n]$ and output $\widehat{\mathbf{s}}[n]$ vectors. Then, the input and output vectors are associated to each other through an LTI relationship. The 
(virtual) analysis filters matrix $\mathbf{H}(z)$ used in the model includes $M^{2}$ filters. Each element $H_{k r}\left(e^{j \omega}\right)$ may be obtained from the $k^{\text {th }}$ analog analysis filter $H_{k}(j \Omega)$ as following [8]:

$$
H_{k r}\left(e^{j \omega}\right)=\frac{1}{M} e^{j \frac{\omega}{M} r} \sum_{m=0}^{M-1} e^{-j \frac{2 \pi}{M} r m} \widetilde{H}_{k}\left(j \frac{\omega}{M}-j \frac{2 \pi}{M} m\right)
$$

where $\widetilde{H}_{k}(j \Omega)$ stands for the periodic extension of $H_{k}(j \Omega)$ considering the interval $\left[-\frac{\pi}{T}, \frac{\pi}{T}\right] . \Omega$ and $\omega$ stand for the frequencies related to the analog and discrete-time signals respectively. It has been shown that all the elements of $\mathbf{H}(z)$ are causal and stable if and only if the analog analysis filters are all causal and stable [8]. According to the practical architecture 2 , there are only $M$ signals $\left[x_{0}[n], x_{1}[n], \ldots\right.$, $\left.x_{M-1}[n]\right]^{T}$ available for reconstructing the original input (the outputs of analysis part).

According to this model, the outputs $\mathbf{x}[n]=\left[x_{0}[n], x_{1}[n]\right.$, $\left.\ldots, x_{M-1}[n]\right]^{T}$ of analysis part may be described in terms of the TDM input vector $\mathbf{s}[n]$ in the frequency domain as following:

$$
\mathbf{X}\left(e^{j \omega}\right)=\mathbf{H}\left(e^{j \omega}\right) \mathbf{S}\left(e^{j \omega}\right)
$$

It is apparently associated with an LTI relationship. The input vector $\mathbf{S}\left(e^{j \omega}\right)$ may be reconstructed through a synthesis stage including the synthesis filters matrix $\mathbf{F}\left(e^{j \omega}\right)$. Therefore, the output vector $\widehat{\mathbf{S}}\left(e^{j \omega}\right)$ can be obtained in the frequency domain as follows:

$$
\widehat{\mathbf{S}}\left(e^{j \omega}\right)=\mathbf{F}\left(e^{j \omega}\right) \mathbf{X}\left(e^{j \omega}\right)=\mathbf{F}\left(e^{j \omega}\right) \mathbf{H}\left(e^{j \omega}\right) \mathbf{S}\left(e^{j \omega}\right)
$$

\section{II-B. Design of synthesis filters matrix}

The TDM architecture of HFB-based ADC is considered (Fig. 2). In the previous section, it was explained that $M$ input samples $s_{0}[n], s_{1}[n], \ldots$, and $s_{M-1}[n]$ are approximated at the output. If one of the analysis or synthesis filters matrices is known, the other one may be calculated. In practice, it is preferable to assume the analog analysis filters a priori regarding to the constraints of analog circuits. Thus, it is here desired to design the synthesis filters assuming a priori $M$ analog circuits as the analysis filters. For conveniently obtaining the synthesis filters matrix $\mathbf{F}\left(e^{j \omega}\right)$, the quantization noise of $\mathrm{A} / \mathrm{D}$ converters is again neglected. Invoking the MIMO model of TDM HFB-based ADC (Fig. 3), the PR equations will be:

$$
\mathbf{F}\left(e^{j \omega}\right) \cdot \mathbf{H}\left(e^{j \omega}\right)=\mathbf{I} \cdot e^{-j \omega n_{d}}
$$

where $\mathbf{I}$ represents the identity matrix $(M \times M)$ and $n_{d}$ stands for an arbitrary delay. $n_{d}$ is considered for holding the causality. Using the Least Squares (LS) optimization, the equation 5 leads to the following solution at each frequency $\omega$ :

$$
\mathbf{F}\left(e^{j \omega}\right)=e^{-j \omega n_{d}} \mathbf{H}^{H}\left(e^{j \omega}\right)\left(\mathbf{H}\left(e^{j \omega}\right) \mathbf{H}^{H}\left(e^{j \omega}\right)\right)^{-1}
$$

where the superscript $(.)^{H}$ stands for the conjugate-transpose operation. This relation may be established for $N$ frequency points ( $N \gg M$ for a suitable interpolation). Thus, the frequency response of each synthesis filter $F_{i j}\left(e^{j \omega}\right)$ can be achieved using (6). A Finite-Impulse Response (FIR) filter may approximate the $(i, j)^{t h}$ element of synthesis filters matrix. Using FIR estimations of synthesis filters, some distortion and interferences may appear. Results may be interpreted in terms of distortion and Inter-Channel Interference (ICI) terms. ICI terms are equivalent for the aliasing terms considered in the classical HFB structure [9]. Supposing FIR synthesis filters, $\mathbf{T}\left(e^{j \omega}\right)$ is defined as following:

$$
\mathbf{T}\left(e^{j \omega}\right)=\mathbf{F}\left(e^{j \omega}\right) \mathbf{H}\left(e^{j \omega}\right)
$$

$\mathbf{T}\left(e^{j \omega}\right)$ is a matrix containing distortion and ICI terms. It shows that the estimated value $\hat{s}_{k}[n]$ of $k^{t h}$ TDM signal $s_{k}[n]$ may be developed in the frequency domain as:

$\hat{S}_{k}\left(e^{j \omega}\right)=\underbrace{T_{k, k}\left(e^{j \omega}\right) S_{k}\left(e^{j \omega}\right)}_{\text {distortion }}+\underbrace{\sum_{m=0, m \neq k}^{M-1} T_{k, m}\left(e^{j \omega}\right) S_{m}\left(e^{j \omega}\right)}_{I C I}$

The $(k)^{t h}$ diagonal element $T_{(k+1)(k+1)}\left(e^{j \omega}\right)$ of $\mathbf{T}\left(e^{j \omega}\right)$ describes the distortion function related to the TDM component $S_{k}\left(e^{j \omega}\right)$. The other $M-1$ elements of $(k)^{t h}$ row of the $\mathbf{T}\left(e^{j \omega}\right)$ represent the related ICI terms. $e^{-j \omega n_{d}}$ is the ideal value of the distortion function and the ICI elements are ideally desired to be null.

\section{SIMULATIONS OF AN EIGHT-BRANCH TDM HFB ADC}

\section{III-A. Implementation of TDM HFB ADC in the time} domain

Using MATLAB/Simulink environment, an eight-branch TDM HFB architecture is simulated in the time domain. A simply-realizable bank of analysis filters is regarded including an RC and 7 RLC circuits. The outputs follow the input signals with a delay. It is reminded that the delay would be $M n_{d} T$ in the TDM HFB case where $M, n_{d}$ and $T$ represent the number of branches, the delay considered at each branch and Nyquist sampling period respectively. It is $M$ times larger than the delay $n_{d} T$ related to the classical HFB. To obtain an acceptable performance with the FIR synthesis filters, a small ratio of each TDM signal spectrum is considered as Guard Band (GB) [8]. Equivalently, an oversampling ratio is used in the classical HFB case. Both the GB and oversampling ratios are here supposed to be $7 \%$. Figure 4 shows the error spectrum when the input is a sinusoidal signal at the frequency $\omega_{\circ}=\frac{0.5 \pi}{8 T}$. For this sinusoidal input, no signal appears at the guard bands of eight TDM components. A parallel to serial operation has been applied to the TDM components for reconstructing the original input. It may be seen that the TDM HFB exhibits clearly a better performance than the classical HFB for this sinusoidal input. An important signal appear at the oversampling spectral area for the classical HFB so that a 


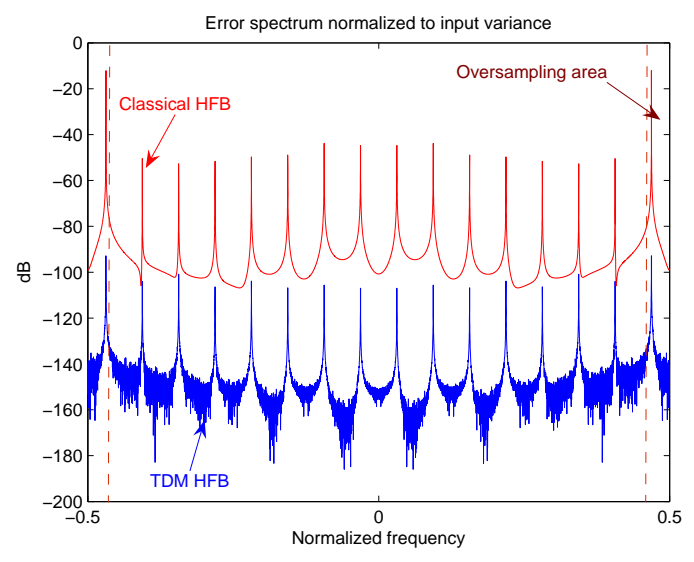

Fig. 4. The error spectrum associated with the TDM (blue) and classical (red) HFB structures for a sinusoidal input.

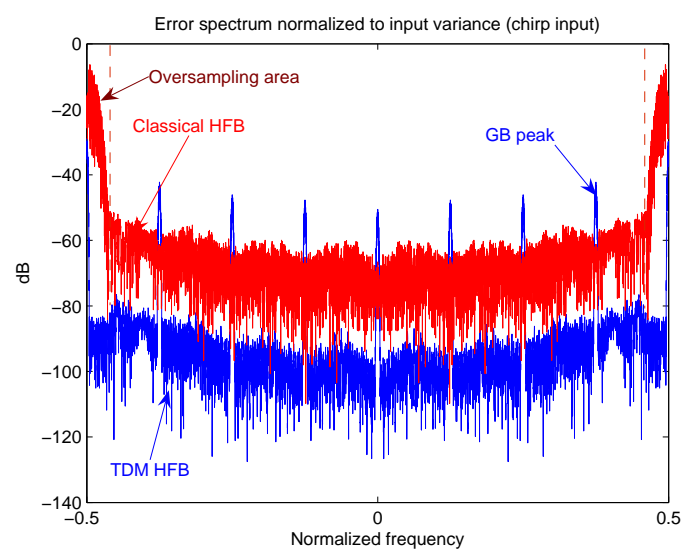

Fig. 5. The error spectrum associated with the TDM (blue) and classical (red) HFB structures for a chirp input.

Post-Filtering (PF) is necessary to omit this part of output signal. For example, Signal-to-Noise Ratio (SNR) at the output of classical HFB structure remains at $49 d B$ without the PF process. If the oversampling spectral area is filtered out, the output SNR would be $73 d B$ for the classical HFB. In return, no signal appears at the guard bands of TDM HFB structure for this sinusoidal input signal. The TDM HFB provides a SNR of $123 d B$ in this case which is $50 d B$ better than the classical architecture. Figure 5 provides a comparison between the TDM and classical HFBs supposing a chirp input signal. The input chirp sweeps the spectrum at the interval $\left[0, \frac{\pi}{T}(1-\alpha)\right]$ that $\alpha$ is supposed to be the oversampling ratio of $7 \%(\alpha=0.07)$. As figure 5 shows, the oversampling area has not been filtered out for the classical HFB neither the GB peaks due to the TDM HFB. Neglecting the oversampling and GB spectral regions, the classical and TDM architectures provide the output SNR of $63 d B$ and $91 d B$ respectively. However, the output of the classical HFB has to be filtered to the frequency interval $[-(1-\alpha) \pi,(1-\alpha) \pi]$. The output of each branch of the TDM HFB is to be post-filtered with the same filter. Thus, the TDM HFB would need $M$ digital filtering process applied to the outputs of $M$ branches. Finally, the simulations in time domain exhibit that the TDM HFB architecture may lead to a better performance than the classical one in the absence of realization errors with respect to the ICI (versus aliasing in the classical case) interference terms.

\section{III-B. Sensitivity to the analog imperfections}

For studying the sensitivity to the realization errors, both the classical and TDM HFB structures are simulated in the presence of analog imperfections. The same eight-branch HFB architectures of the previous section are considered. To observe the effects of realization errors, all electronic elements ( $\mathrm{R}, \mathrm{C}$ and $\mathrm{L}$ ) included in the analysis filter bank are considered with a Gaussian profile. The STandard Deviation (STD) of Gaussian distribution is employed for representing the analog imperfections. The simulations are repeated for 1000 trials of each value of realization error. The output resolution of HFB structures are used for comparison. Firstly, the input is assumed to be a sinusoidal signal at the frequency $\omega_{\circ}=\frac{0.5 \pi}{8 T}$. Figure 6 shows the output resolution (in bits) of the classical and TDM HFBs versus the realization errors. If

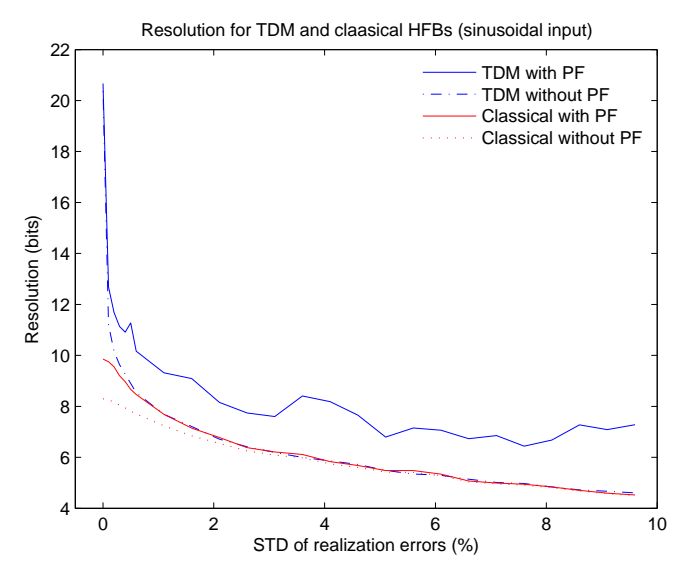

Fig. 6. The output resolution of the classical (in red) and TDM (in blue) HFB architectures versus the relative realization errors. A sinusoidal signal has been applied to the input.

Post-Filtering (PF) is applied for eliminating the oversampling and GB spectral areas in the classical and TDM cases respectively, the TDM HFB architecture is associated with a performance of 3 bits better than the one related to the classical HFB in the presence of realization errors. It means that the TDM HFB is less sensitive than the classical one to the realization errors in the case of sinusoidal input. In other words, the SNR at the output of this eight-branch TDM HFB is about $20 d B$ better than the one related to the classical 
HFB. If GB spectral areas are not filtered out in the TDM HFB, it leads to the same resolution that a classical HFB may provide with eliminating the oversampling band. This shows that the TDM HFB architecture may provide at worst case (meaning without PF) the same performance that the classical one. To have a comparison in the whole spectrum, a chirp input sweeping the frequency interval $\left[0, \frac{\pi}{T}(1-\alpha)\right]$ is applied to both the TDM and classical structures. $\alpha$ stands for the oversampling ratio of $7 \%(\alpha=0.07)$. Like to the sinusoidal case, a similar procedure is applied to obtain the sensitivity to the realization errors. Figure 7 illustrates the output resolution of TDM and classical HFBs versus the STD of realization errors. For the chirp input signal,

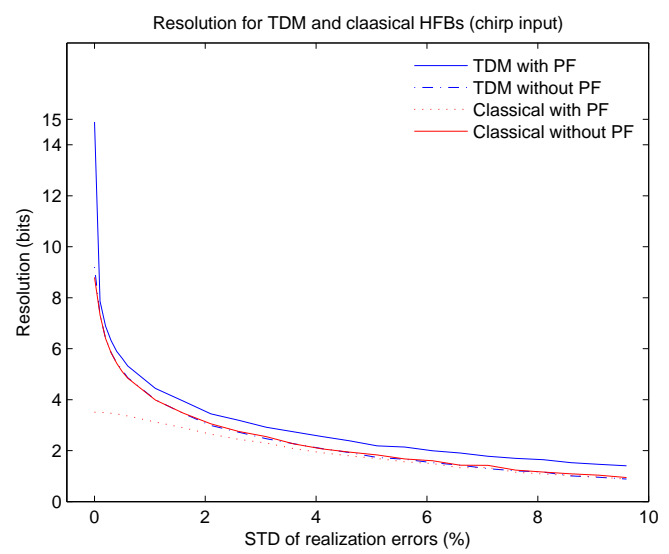

Fig. 7. The output resolution of the classical (in red) and TDM (in blue) HFB architectures versus the relative realization errors. A chirp signal is considered as the input.

the TDM HFB architecture exhibits a better performance about 1 bit in the presence of analog imperfections than the classical HFB. It is reminded that the performance of TDM HFB is much better than the one related to the classical HFB in the absence of realization errors (refer to Fig. 7 at the STD of errors equal to zero). Another interesting result may be deducted from these two simulations. According to figures 6 and 7, the TDM HFB may provide a performance approximately equal to the classical HFB even if no PostFiltering (PF) is considered to eliminate the GB spectral areas. However, if the oversampling spectral area is not postfiltered out in the classical HFB, the performance degrades so much.

\section{CONCLUSION}

The simulations of TDM HFB-based ADCs in the time domain (using MATLAB/Simulink) show that the mathematical equations for the TDM architecture in the frequency domain are valid in the time domain. It is also shown that the TDM architecture may provide much better performance in terms of the output resolution than the classical HFB in the absence of realization errors (about 10 and 6 bits improvement for the sinusoidal and chirp inputs respectively). In the presence of realization errors, the TDM HFB leads to a larger resolution ( 3 and one bits) than the classical one (for the sinusoidal and chirp inputs respectively). The Post-Filtering process seems to always be necessary for the classical HFB for eliminating the oversampling spectral area. Although the TDM HFB requires $M^{2}$ digital synthesis filters compared to $M$ ones of classical case, the computation complexity per each output sample is the same for both structures because the TDM HFB provides $M$ output samples at each cycle. Finally, an LTI relationship governs between the input and outputs of the TDM HFB but it is not LTI for the classical case. Thus, a blind deconvolution may be applied only to the TDM architecture for adaptively correcting the realization errors. This is not possible for the classical HFB-based ADC.

\section{REFERENCES}

[1] Schreier R., Temes G.C., ”Understanding Delta-sigma data converters", Wiley-IEEE Press, 2004.

[2] Tuttlebee W., Manor R., "The Software radio technology: a european perspective", IEEE Communications Magazine, Feb., 1999, pp.118-123.

[3] Franca J., Petraglia A., Mitra S. K., "Multirate AnalogDigital systems for signal processing and conversion ", Proceedings of the IEEE, vol.65, no.2, Feb., 1997, pp.242-262.

[4] Velazquez S.R., Nguyen T.Q., Broadstone, S.R., "Design of Hybrid Filter Banks for Analog/Digital conversion", IEEE Transactions On Signal Processing, vol.46, no.4, Apr., 1998, pp.956-967.

[5] Asemani D., Oksman J., "Influences of oversampling and analog imperfections on hybrid filter bank A/D converters", IEEE MWSCAS, San Juan, Puerto Rico, Aug., 2006.

[6] Sanada Y., Ikehara M., "Digital compensation scheme for coefficient errors of complex filter bank parallel A/D converter in low-IF receivers ", Proceedings of 55th IEEE Vehicular Technology Conference, vol. 4, May, 2001, pp.1680-1684.

[7] Pinheiro M. et. al., "Improving the near-perfect HFB performance in the presence of realization errors", ICASSP, 2001, vol. 2, pp.1069-1072.

[8] Asemani D., Oksman J., "Time-Division Multiplexing architecture for Hybrid Filter Bank A/D converters", submitted to IEEE MWSCAS, Montreal, Canada, Aug., 2007.

[9] Lowenborg P., Johansson H., Wanhammar L., "Twochannel digital and hybrid Analog/Digital multirate filter banks with very low-complexity analysis or synthesis filters", IEEE Transactions on circuits and Systems, vol.50, no.7, July, 2003, pp.355-367. 